\title{
Evaluación Bidireccional del Proceso Enseñanza-Aprendizaje Universitario Aplicando Análisis de Componentes Principales
}

\section{Borja Velázquez-Martía ${ }^{*}$, Juan José Pérez-Arévalo ${ }^{b}$}

a Departamento de Ingeniería Rural y Agroalimentaria, Universidad Politécnica de Valencia (Spain), borvemar@dmta.upv.es, ${ }^{b}$ Facultad de Ingeniería, Universidad Católica de Santiago de Guayaquil (Ecuador), obras.civiles.perez.perez@gmail.com.

\begin{abstract}
The evaluation of education systems in most universities is based on the analysis of the exclusive assessment of the students, which are subject to some surveys where they are asked about their perceptions on various aspects of the organization, methodology, motivation raised by the teacher, and evaluation system. However, there may be discrepancies between the perception of teachers and students, which are not sometimes considered in the evaluation processes of the system. This paper proposes the hypothesis that the teachinglearning process is positively developed if it is simultaneously highly valued by both groups. The applicability of Principal Component Analysis as method of assessment of the appreciation of educational process based on surveys of teachers and students has been demonstrated, and it allows to identify the need for corrective actions.
\end{abstract}

Keywords: teaching methods, teaching evaluation, active methodologies; training assessment

\footnotetext{
Resumen

En la mayoría de las universidades la evaluación del sistema de enseñanza se basa en el análisis de la apreciación exclusiva de los alumnos, los cuales son sometidos a unas encuestas, donde se les pregunta sobre su percepción en distintos aspectos como la organización, metodología, motivación que suscita el profesor y el sistema de evaluación. Sin embargo, pueden haber discrepancias entre la percepción de los profesores y estudiantes que a veces no son consideradas en los procesos de evaluación del sistema. Este trabajo propone la hipótesis de que el proceso de enseñanza-aprendizaje se desarrolla positivamente en cuanto es bien valorado simultáneamente por ambos colectivos. Se demuestra la aplicabilidad del Análisis de Componentes Principales como técnica de evaluación de la apreciación de las técnicas educativas a partir de los valores dados a las respuestas de una serie de encuestas a docentes y estudiantes, y permite identificar la necesidad de medidas correctivas.
}

Palabras clave: métodos docentes, evaluación enseñanza, metodologías activas, evaluación educativa. 


\section{Introducción}

El éxito de la educación depende de muchos factores, entre ellos, la organización, las metodologías de trabajo, la motivación y la evaluación (Mourshed, Chijioke, y Barber, 2010). Por ello, el análisis de estos factores nos permite aprender a promover sistemas eficientes, corrección de errores, y orientar los procesos de enseñanza-aprendizaje (Sueiro, 2014).

Zabalza (2004), afirma que el aprendizaje de los estudiantes depende en gran medida de sus capacidades, esfuerzo e interés, pero también obedece a que hayan tenido buenos maestros, buenos recursos. Ello obliga al análisis del contexto en el que se realiza el proceso (Vasilachis de Gialdino, 2009). La adaptación del proceso de enseñanza al contexto supone un reto para el docente (Vélaz de Medrano y Vaillant, 2009; Jover, 2011).

En la mayoría de las universidades la evaluación de los sistemas de enseñanza se basa en la apreciación exclusiva de los alumnos, los cuales son sometidos a unas encuestas donde se les pregunta sobre su percepción sobre distintos aspectos como la organización, metodología, motivación que suscita el profesor y el sistema de evaluación. Sin embargo, es bastante común una sorpresa del profesor evaluado, cuando habiendo obtenido una evaluación baja o media, su impresión había sido significativamente diferente durante el curso. En este trabajo se propone la hipótesis de que el proceso de enseñanza-aprendizaje se desarrolla positivamente en cuanto es bien valorada simultáneamente tanto por los estudiantes como por los profesores. Una evaluación coincidente entre docentes y estudiantes garantiza la excelencia del proceso. Por una parte, el profesor, experto en la materia que imparte, tiene una cosmovisión mucho más amplia que la de los alumnos, eso le permite elaborar la estrategia y fijar los objetivos adecuados del proceso de enseñanza aprendizaje. Por otra parte, el alumno posee de antemano unas expectativas de aprendizaje que deben ser satisfechas.

El análisis de la percepción simultánea de profesores y estudiantes no ha sido tratado con suficiente profundidad. Según Zabalza (2012), en las dos últimas décadas la investigación sobre las metodologías que el profesorado utiliza, así como los aspectos referidos a la motivación del alumnado, han sido tema de considerable interés y discusión en el campo de la educación.

La manera habitual de evaluar la percepción que tienen los alumnos del proceso de enseñanza-aprendizaje dirigido por un profesor es la realización de una encuesta antes de la evaluación final de la materia. De esta manera el resultado de la encuesta no queda condicionado por la situación siempre conflictiva de calificación del alumno por una nota alfanumérica, de 0 a 10 en países latinos, o A ,B, C o D en países anglosajones. La discrepancia en la calificación de los alumnos suele ser común debido a matices de percepción.

En este trabajo se propone como sistema de evaluación del sistema de enseñanza la realización de la misma encuesta bidireccional a los estudiantes y a los docentes. Para

(cc) EY-NG-ND 2016, Universitat Politècnica de València 
contrastar resultados se proponen métodos estadísticos basados en Análisis de Componentes Principales (ACP). Esta encuesta debe ser centrada en aspectos generales del proceso, no atendiendo a destrezas particulares de alumnos y profesores. Hay que tener en cuenta aspectos muy importantes que centran la responsabilidad del alumno, por ejemplo: De Miguel-Díaz (2005) sostiene que uno de los objetivos principales en la educación europea es promover un proceso de renovación de la metodología en la enseñanza superior, centrado en el aprendizaje independiente del estudiante, frente al tradicional centrado en el docente. Esta metodología en la que el estudiante asume la responsabilidad de su aprendizaje implica sentar bases distintas:

Que los docentes se amolden a los nuevos conocimientos, tipologías patrones culturales, avances tecnológicos, valores dominantes..., que se originan en la sociedad del conocimiento y la información, implicando un proceso de constante actualización, para lo cual cada docente requiere de una preparación personal que le ayude a la interpretación de la información aprovechable y la generación de su propio conocimiento de forma continua.

Los estudiantes deben comprender que el proceso formativo es responsabilidad personal, y él mismo asume el protagonismo. De ahí que uno de los objetivos de la educación sea infundir en los estudiantes su forma de aprender a lo largo de toda su vida.

Debe asumirse la renovación de las metodologías docentes en la enseñanza superior, orientándose al fortalecer el estudio independiente para que el egresado pueda incorporarse a sus tareas profesionales de forma brillante y responsable (Sáez, 2000).

García-Benau, Sierra-García, y Zorio-Grima (2012) afirman que tradicionalmente la universidad se ha encargado de suministrar a los estudiantes conocimientos fundamentalmente teóricos basados en la conferencia magistral. Sin embargo, la Unión Europea (UE), para homologación de las titulaciones en Europa, esboza una enseñanza centrada en el aprendizaje, focalizada en el desarrollo de competencias, destrezas y habilidades, requeridos para el ejercicio profesional. Se observa asimismo que uno de los aspectos contemplados en esas prioridades dentro de este espacio europeo son las metodologías docentes. El cambio de paradigma en la educación supone indagar en nuevos sistemas de organización del trabajo, técnicas de enseñanza y evaluación. Este nuevo marco docente que se vislumbra se caracteriza por un claro cambio en el modelo de enseñanza. La universidad debe facilitar al estudiante los instrumentos para que aprenda, y de manera especial, para que aprenda a investigar (Luengo-Navas, Luzón-Trujillo, y Torres-Sánchez, 2008). La pregunta que se suscita en la comunidad científica es cómo influyen distintos cambios a la percepción de alumnos y profesores en el proceso de enseñanza-aprendizaje. Antón-Ares (2005) señala que el desarrollo de sistemas de análisis continuos pasa a ser muy relevante y un elemento imprescindible para mejorar la calidad de la educación universitaria. $\mathrm{Y}$ en este mismo sentido, Tejedor-Tejedor (2003) argumenta que un sistema de evaluación del sistema no debe presumir de la concentración de una sucesión de pruebas llevadas a cabo de manera puntual, sino de un proceso continuo y sistemático de forma que permita el perfeccionamiento docente y la planificación de las clases y se ajuste a las necesidades del claustro, profesores, estudiantes y la comunidad. Guba y Lincoln (1989) afirman que la meta 
primordial de la ciencia social es la interpretación de fenómenos dentro de su contexto y no la generalización.

\section{Objetivos}

El objetivo principal de este trabajo ha sido proponer un sistema de evaluación del sistema de enseñanza aprendizaje mediante la aplicación Análisis de Componentes Principales a un pase de encuestas bidireccional, tanto a estudiantes como profesores, para detectar discrepancias entre la percepción de los mismos. Este trabajo propone la hipótesis de que el proceso de enseñanza-aprendizaje se desarrolla positivamente en cuanto es bien valorado simultáneamente por ambos colectivos.

Un objetivo secundario de este trabajo es buscar la relación que existe entre las dimensiones/categorías: organización, metodología, evaluación y motivación, como una herramienta que nos permite estudiar en forma ordenada la realidad educativa, que ayudará al docente a desarrollar su práctica profesional y lograr adecuadas prácticas docentes con los mejores procesos en el proceso enseñanza-aprendizaje que se traducirán en el rendimiento académico de los alumnos (Sánchez-García, García-Chamizo, Mora-Mora, y Signes-Ponts, 2007).El presente trabajo pretende determinar cómo influyen distintos aspectos del proceso enseñanza-aprendizaje, tales como la organización en el aula, el uso de las metodologías, como las TIC, y el uso de las distintas formas de evaluación en la motivación en el aprendizaje de los estudiantes en las carreras de arquitectura e ingeniería de la Universidad Católica Santiago de Guayaquil (UCSG), es decir, el grado de satisfacción de los estudiantes y profesores, dentro del proceso docente educativo de las dos carreras.

\section{Materiales y métodos}

Esta investigación se ha realizado en el área de las asignaturas técnicas de las carreras de arquitectura e ingeniería de la Universidad Católica de Santiago de Guayaquil (UCSG). Las fuentes para tomar datos de la población a estudiar se basó en tres técnicas: el pase de encuestas, la realización de entrevistas y análisis de documentos: Planes de estudio - Guía docente (Syllabus).

La encuesta contaba con 69 preguntas, con la intención de que tanto el profesorado como el alumnado realicen una evaluación la gestión en el aula, de las metodologías y evaluación de las asignaturas, y si éstas motivan o no en el aprendizaje de los estudiantes. El cuestionario contiene las mismas preguntas tanto para los estudiantes como para los docentes de las dos carreras. Las preguntas estaban clasificadas por cuatro categorías o dimensiones, y subcategorías mostradas en la Tabla 1.

Dichos instrumentos se estructuraron en formato de Escala Likert con cuatro opciones de respuesta: Nunca $=1 ;$ A veces $=2$; Casi siempre $=3$; y Siempre $=4$ 
Para la validación del contenido del cuestionario a los docentes y estudiantes, se eligió cinco doctores por su excelente carrera profesional con el fin de que puntuaran de 0 a 5 cada uno de los ítems de los cuestionarios, atendiendo a los siguientes criterios:

Calidad técnica: hace referencia a la calidad del lenguaje, es decir al grado en el que el lenguaje utilizado es el adecuado y no presenta sesgos que puedan inducir a confusión.

Coherencia: se refiere a la relación entre el ítem y la dimensión que pretende medir.

Representatividad: hace referencia a la bondad de los ítems, en cuanto éstos permiten valorar la motivación para el aprendizaje de los estudiantes, con las metodologías con las que imparten las profesoras y profesores sus asignaturas.

Tabla 1. Categorización de las entrevistas.

\begin{tabular}{|c|c|c|}
\hline No. & Categorías & Subcategorías \\
\hline 01 & Organización en el aula & $\begin{array}{l}\text { 1. Organización del aula. } \\
\text { 2. Como organizan y trabajan los docentes con sus alumnos. }\end{array}$ \\
\hline 02 & Metodologías de estudio & $\begin{array}{l}\text { 1. El modo como el docente organiza a sus alumnos. } \\
\text { 2. De las metodologías, entre ellas las Tics. } \\
\text { 3. De la ejecución de las tareas. } \\
\text { 4. Ante las posibles dificultades. } \\
\text { 5. Del trabajo con sus alumnos. } \\
\text { 6. De las tareas con sus alumnos. }\end{array}$ \\
\hline 03 & Estrategias de motivación & $\begin{array}{l}\text { 1. Motivación del alumnado. } \\
\text { 2. De la explicación a sus alumnos. } \\
\text { 3. Al encargar una tarea para la casa a sus alumnos. } \\
\text { 4. Del proceso para resolver las tareas. }\end{array}$ \\
\hline 04 & Estrategias de evaluación & $\begin{array}{l}\text { 1. El uso de las distintas formas de evaluación. } \\
\text { 2. De las metas y objetivos que se persiguen. }\end{array}$ \\
\hline
\end{tabular}

Aquellos que obtuvieron un índice superior a 2,5 en cada uno de los ítems se incluyeron en la versión definitiva de los cuestionarios.

Para medir la consistencia interna y fiabilidad del instrumento de medida (cuestionario) se utilizó coeficiente alfa de Cronbach para lo cual se realizó una prueba piloto en las dos escuelas. Este coeficiente alcanzó los siguientes valores; arquitectura: docentes 0,873, estudiantes 0,943 ; ingeniería civil: docentes 0,914 , estudiantes 0,930 . Valores que aseguran un alto grado de fiabilidad sobre las respuestas de los docentes y de los estudiantes de las dos carreras, y verifican las recomendaciones de los expertos (George y Mallery, 2003).

Para las entrevistas a los docentes se elaboraron 20 preguntas, sobre la forma como el profesor organiza, desarrolla y evalúa el curso, y su desempeño como docente. Éstas fueron semi-estructuradas para facilitar su manejo, en razón de obtener la información pertinente al objetivo específico de este estudio, esto es, en lo relacionado a como el profesorado entiende la metodología que emplea en las clases, y cómo influye en la motivación del alumnado para su aprendizaje (UNE-66181:2008, 2008). Las preguntas de las entrevistas se clasificaron también por categorías o dimensiones según la Tabla 1.

\subsection{Población y muestra}

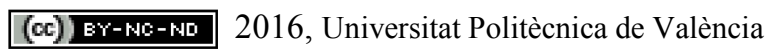

Congreso In-Red (2016) 
Para la selección de los informantes se siguió un proceso de selección de la muestra de carácter intencional-opinático. La muestra fue el 100\% de los docentes de cada una de las escuelas y el $100 \%$ de los estudiantes de los últimos ciclos de estudio tanto del semestre A como del semestre B en el año 2014. De los 50 encuestados docentes (100\%) en una de las escuelas 7 no entregaron las encuestas, por lo que se trabajó con 43. En razón de lo cuál, la muestra fue 43 docentes y 43 alumnos, tanto en la carrera de arquitectura como en la de ingeniería civil. En las entrevistas participaron el 20\% de los docentes encuestados, es decir, 10 profesores por cada escuela, tomados al azar, aunque tratando que sean o hayan sido Directivos o con muchos años de experiencia en la docencia.

\subsection{Análisis estadístico}

Para cada encuestado se identificaron el sexo, el rol desempeñado en el proceso enseñanzaaprendizaje (estudiante/docente) y la carrera técnica a la que está vinculado (arquitectura/ingeniería). Dado que las 60 preguntas estaban estructuradas en cuatro categorías y las respuestas estaban valoradas de 1 a 4, según se ha indicado (Nunca = 1; A veces $=2$; Casi siempre $=3$; y Siempre $=4$ ), los valores obtenidos en cada una de las preguntas asociadas a cada una de las categorías fueron sumados. De este modo cada encuestado tiene asociadas cuatro variables o dimensiones, que son los valores obtenidos en cada categoría. Para analizar si existe influencia de los factores sexo, rol y estudios a los que está vinculado el encuestado, se realiza un análisis de componentes principales.

Este mismo proceder puede ser seguido para el análisis de las subcategorías que son 14, de forma que a cada individuo se le asocian 14 variables dimensiones numéricas.

Por otro lado se realizaron tablas de contingencia para determinar si existen diferencias significativas en las cuestiones realizadas en cada categoría, a través de análisis basado en la distribución Chi-cuadrado.

\section{Resultados y Discusión}

\subsection{Análisis de las componentes principales}

Dado que cada individuo estaba calificado por cuatro variables o dimensiones numéricas, una por categoría, (puntuación obtenida de la suma de los valores de sus respuestas en las preguntas de cada categoría), se ha analizado la correlación lineal de los valores obtenidos a través del coeficiente de correlación de Pearson. El rango de estos coeficientes de correlación va de -1 a +1 y miden la fuerza de relación lineal entre las variables o dimensiones. Un valor cercano a +1 significa que existe una relación lineal positiva, es decir, si aumenta el valor de una variable o dimensión también lo hace la otra. Si el valor está cerca de -1 , la relación es lineal negativa, es decir, cuando una variable aumenta la otra disminuye. Si el coeficiente de Pearson está cercano a 0 , significa que no hay relación entre las variables. Se puede observar en la Tabla 2 que todas las categorías tienen correlaciones positivas. Sin embargo, hay unas

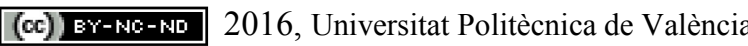


relaciones más fuertes y otras más débiles. La percepción positiva de la metodología de trabajo se correlaciona mucho con la percepción tenida en la motivación y evaluación dado que se obtienen coeficientes de Pearson muy cercanos a la unidad $(0,85$ y 0,82$)$. Sin embargo, se puede observar que la organización dentro del aula, aunque también tiene efectos positivos, no influye tanto en estos aspectos, dado que su correlación con la motivación y evaluación es sólo de 0,51 y 0,41 . Se puede observar que aunque se tenga una buena organización, la percepción metodológica no tiene porqué ser buena globalmente (tanto en estudiantes como en docentes, y considerando conjuntamente las dos escuelas), su coeficiente de Pearson es de 0,56 . Se evidencia que la motivación positiva se relaciona mucho también con la evaluación positiva 0,83 .

Tabla 2. Coeficientes de correlación de Pearson entre las categorías

\begin{tabular}{ccccc}
\hline Variables & Organización & Metodología & Motivación & Evaluación \\
\hline Organización & 1 & 0,560 & 0,510 & 0,411 \\
Metodología & 0,560 & 1 & 0,848 & 0,823 \\
Motivación & 0,510 & 0,848 & 1 & 0,833 \\
Evaluación & 0,411 & 0,823 & 0,833 & 1 \\
\hline
\end{tabular}

El Análisis de Componentes Principales (ACP) es una técnica basada en generar a partir de las variables o dimensiones asociadas a los individuos de la muestra, ecuaciones con el mejor coeficiente de determinación R2 posible. Cada una de las ecuaciones se denomina componente. Si analizamos los dos componentes con mejor R2 podemos representar cada uno de los datos en un gráfico de dispersión. En Tabla 3 se muestran los coeficientes de los componentes principales (ecuaciones). Por ejemplo, el primer componente principal F2 tiene la ecuación (1).

F1 $=0,5181 *$ Evaluación $+0,5411 *$ Metodología $+0,536208 *$ Motivación $+0,3891 *$ Organización

El segundo componente principal F2 tiene la ecuación (2).

F2 $=-0,372 *$ Evaluación $-0,099 *$ Metodología $-0,195 *$ Motivación $+0,902 *$ Organización

Donde los valores de las variables en la ecuación están estandarizados, substrayendo sus medias y dividiéndolos por sus desviaciones típicas.

Tabla 3. Coeficientes de las ecuaciones ( 1 y 2 ) que forman cada componente

\begin{tabular}{ccccc}
\hline & F1 & F2 & F3 & F4 \\
\hline Organización & 0,389 & 0,902 & 0,171 & $-0,074$ \\
Metodología & 0,541 & $-0,099$ & $-0,384$ & 0,742 \\
Motivación & 0,536 & $-0,195$ & $-0,480$ & $-0,666$ \\
Evaluación & 0,518 & $-0,372$ & 0,770 & $-0,029$ \\
\hline
\end{tabular}

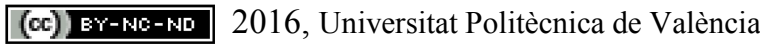

Congreso In-Red (2016) 
En la Figura 1 se muestra el gráfico de dispersión de los datos obtenidos de la aplicación de las ecuaciones de los componentes principales, junto a la caracterización de cada encuestado según sea estudiante o docente. En conjunto las dos primeras componentes explican el $92,24 \%$ de la variabilidad de la población en sus respuestas.

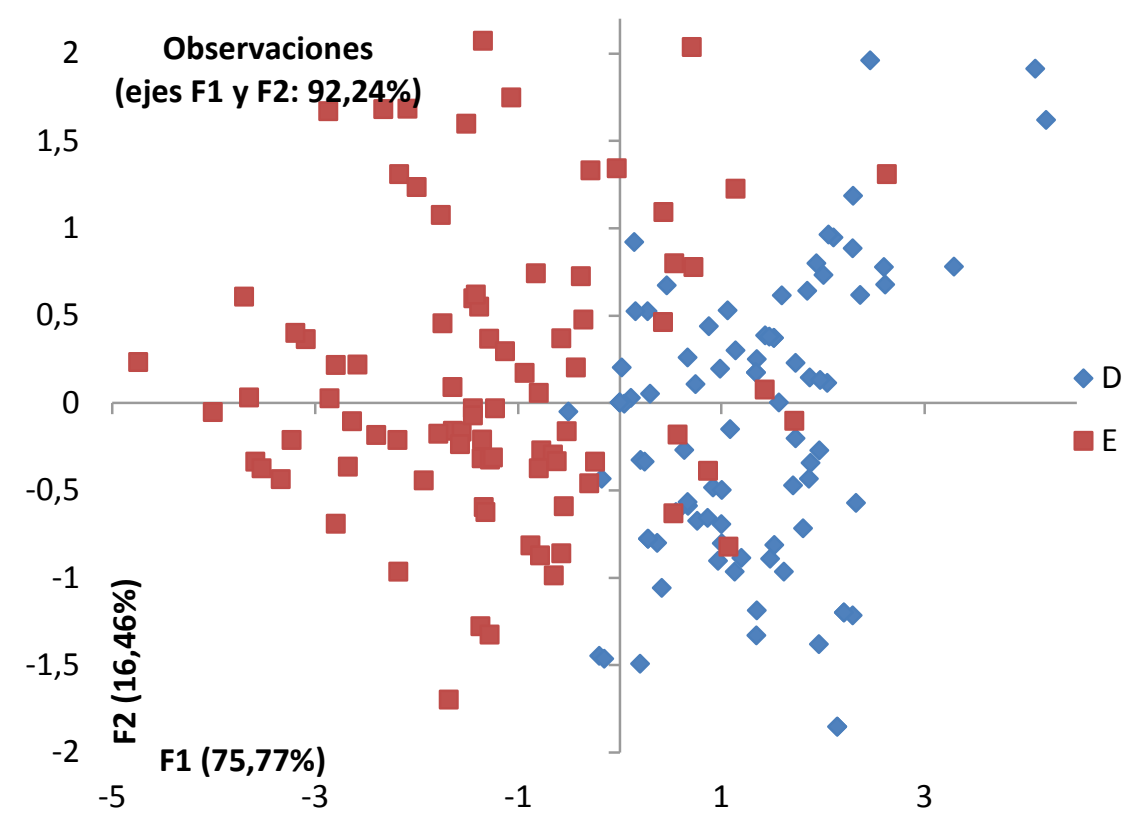

Fig. 1. Dispersión de los datos aplicando las ecuaciones de componentes principales (ACP) identificando los encuestados según rol en el proceso enseñanza-aprendizaje; D: Docente, E: estudiante.

Como se puede observar en la Tabla 3 el componente F1 está influenciado mayoritariamente por la percepción de los encuestados en la Metodología, Motivación y Evaluación. Sin embargo en F2 el mayor peso recae en la Organización, siendo los coeficientes de la Metodología, Motivación y Evaluación negativos. Esto significa que los encuestados con una percepción muy alta de la Organización estarán por la parte superior de la gráfica de la Figura 3. Los encuestados con una percepción mala de la Organización estarán por la parte inferior de la gráfica. Según la ecuación F1 los encuestados que tengan una valoración muy positiva conjuntamente en las categorías Metodología, Motivación y Evaluación estarán muy a la derecha de la gráfica. Los encuestados que tengan una percepción conjunta baja en las mismas estarán muy a la izquierda de la gráfica. Se puede observar en la Figura I que la población de docentes y estudiantes tienen una percepción significativamente diferente en cuanto a Metodología, Motivación y Evaluación. Los estudiantes se agrupan mayoritariamente a la izquierda de la gráfica, mientras que los profesores se agrupan mayoritariamente a la derecha de la gráfica.

Si representamos la misma gráfica identificando cada individuo por sus estudios de ingeniería o arquitectura (Figura 2), se observa que los encuestados de cada titulación se distribuyen de forma homogénea, no formando grupos diferenciados. Esto significa que no hay diferencias de opinión según la carrera en la que el encuestado está involucrado, ya sea docente o estudiante. 


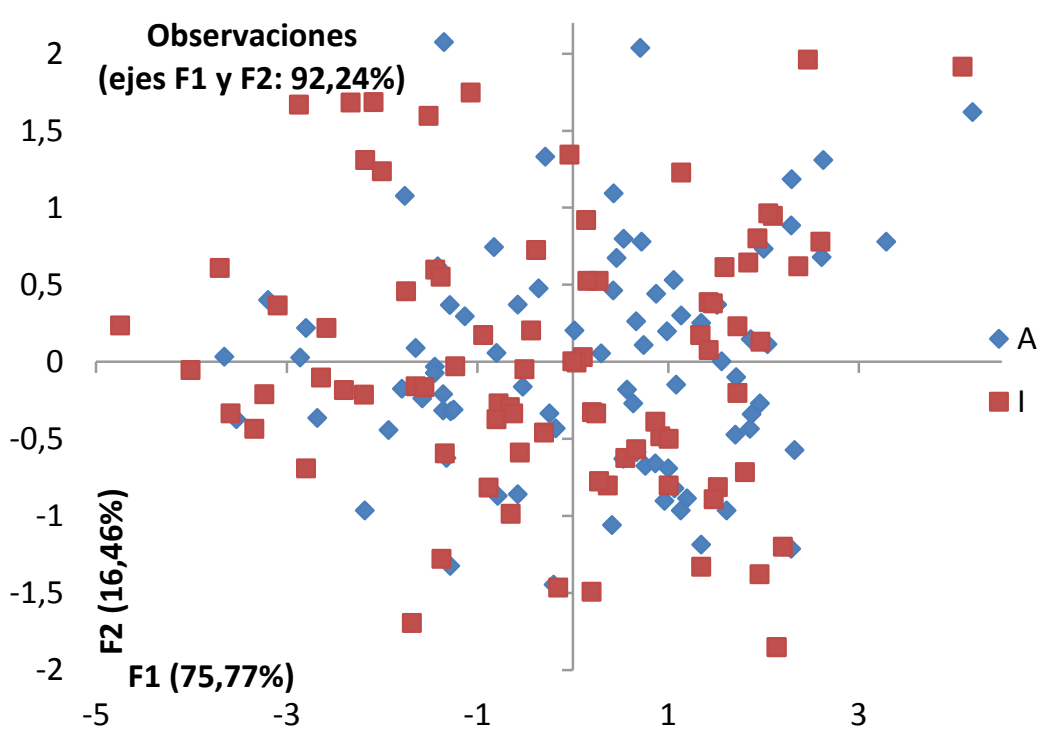

Fig. 2. Dispersión de los datos aplicando las ecuaciones de componentes principales (ACP) identificando los encuestados según titulación I: ingeniería, A: arquitectura

La dispersión de la Figura 3 de los encuestados según el sexo (Hombres y Mujeres), están uniformemente mezclados de tal manera que no hay diferencias significativas entre la percepción de éstos.

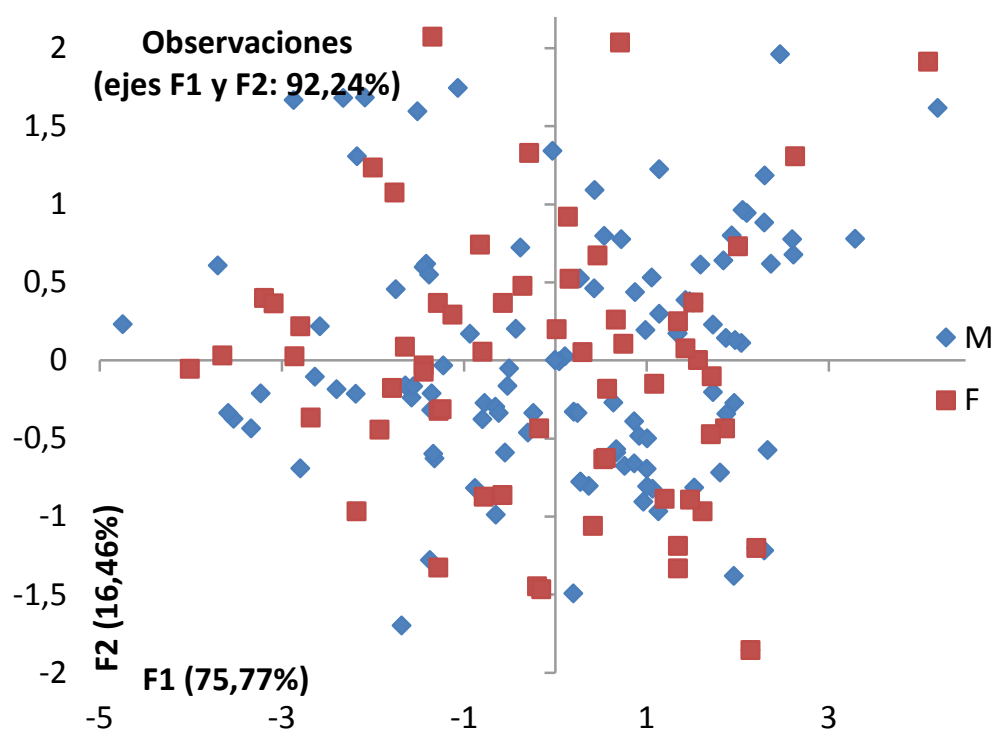

Fig. 3. Dispersión de los datos aplicando las ecuaciones de componentes principales (ACP) identificando los encuestados según sexo F: femenino; M: masculino. 


\subsection{Análisis de las tablas de contingencia}

Estudiando la contingencia de cada una de las sub-categorías estudiadas, es decir, los porcentajes de cada uno de los valores obtenidos en las respuestas de los docentes y estudiantes de arquitectura e ingeniería en cada una de las categorías (organización del trabajo en el aula; metodologías de trabajo; estrategias de motivación y estrategias de evaluación), donde se evalúa la percepción que tienen los profesores y los estudiantes en cada una de ellas.

Se observa que la percepción de los profesores de arquitectura respecto a su organización del aula es muy positiva (Subcategoría 1). Prácticamente más del $90 \%$ piensan que facilita espacios para la discusión con sus estudiantes. Sin embargo, el 30\% indica que nunca o casi nunca trabaja con sus estudiantes en forma individual, ni en parejas. El 95\% manifiesta que nunca trabaja con grupos de tamaño medio. El 50\% indica que siempre o casi siempre trabaja con grupos grandes.

Es muy llamativo que la percepción de los estudiantes es significativamente diferente. Más del $60 \%$ opina que no se facilitan espacios para la discusión con sus estudiantes. Habría que hacer un análisis de cuales son los motivos de esta discrepancia. El 50\% de los estudiantes piensa que sólo a veces o nunca la organización del aula permite discutir sobre diferentes temas. Más del $75 \%$ de los estudiantes perciben que nunca o casi nunca se trabaja de forma individualizada. Este hecho también es corroborado por los docentes. Quizás podría mejorarse la generación de espacios de discusión trabajando este aspecto. Se muestra la misma tendencia de repuestas en los estudiantes de ingeniería que en los estudiantes de arquitectura.

Es de destacar que el 98\% de los docentes indican que durante la ejecución de las tareas se ponen de manifiesto los errores y se indica cómo pueden corregirlos; sin embargo más del $50 \%$ de los estudiantes no lo perciben así. En la escuela de ingeniería tanto los docentes como los discentes opinan de manera similar. Por tanto, habría que analizar el empeño que ponen los profesores en este aspecto, o la falta de apreciación por parte de los estudiantes.

En las respuestas de los docentes en las preguntas vinculadas a la categoría 2 relacionada con metodologías aplicadas se observa que piensan que siempre o casi siempre se indica el modo en que hay que organizarse para el correcto proceso de aprendizaje en las diferentes materias. Sin embargo, más del 30\% de los estudiantes discrepan con esta opinión. ¿Es esto tolerable o asumible? Lo mismo se observa cuando se plantea la cuestión de si los profesores priorizan en sus clases las actividades de exposición, más del 70\% de los estudiantes piensan que sí se realiza esta priorización, sin embargo un 30\% muestran desacuerdo. El 80\% de los docentes de arquitectura piensan que siempre o casi siempre en sus clases establecen el dinamismo suficiente para que los alumnos no se aburran. Sin embargo el $60 \%$ de los alumnos opinan que esto se consigue a veces o nunca.

(cc)) EY-NG-ND 2016, Universitat Politècnica de València 
En relación a la subcategoría 4 (metodologías docentes entre ellas las TIC), el 82\% de los docentes y estudiantes de arquitectura coinciden en que se utilizan dispositivos audiovisuales en el aula: proyector, televisor entre otros. En la escuela de ingeniería también coinciden en esto algo más del $70 \%$ de los docentes y estudiantes. Sin embargo, en relación a si se emplea materiales impresos diferentes al libro de texto, los docenes de arquitectura responden en un $60 \%$ que casi siempre y siempre dan materiales diferentes al libro de texto, sin embargo esto lo opinan sólo alrededor del 40\% de los estudiantes. Esta diferencia de opinión aumenta un poco entre los docentes y estudiantes de ingeniería, siendo el 70\% y 40\%, respectivamente. Esto nos permite concluir que ciertas tecnologías audiovisuales sí están implantadas en las aulas, pero que el trabajo personal de los estudiantes sigue basándose mayoritariamente en la utilización de un solo libro de texto, infrautilizándose otros medios de información como internet, bases de datos, artículos científicos, revistas especializadas, trabajos de prospección, etc.

Es importante observar las respuestas relacionadas con la categoría estrategias de motivación, en la que el $85 \%$ de los profesores afirman que señalan los objetivos que alcanzarán al finalizar el tema, pero el $45 \%$ de los alumnos indican que esto los docentes no lo hacen nunca o sólo a veces.

Se puede observar, en lo que se refiere a la categoría estrategias de motivación, la percepción de los docentes es diferente a la de los estudiantes, por lo que se deberá poner mucha atención en el esfuerzo y empeño que ponen los docentes por lograr motivar a sus estudiantes. Prácticamente en todas las cuestiones contempladas los docentes manifiestan valores de siempre o casi siempre, mientras los estudiantes manifiestan esos valores alrededor del $50 \%$.

En lo que se relaciona a la categoría estrategias de evaluación, se observa que hay la misma tendencia en la apreciación que las manifestadas en las categorías metodologías de estudio aplicadas y estrategias de motivación, existiendo diferencias significativas entre docentes y estudiantes. En lo referente a la consulta a los docentes si creen que el sistema de evaluación que usan detecta el grado de adquisición de conocimientos de sus alumnos sobre la asignatura tratada, éstos responden en un $98 \%$ que casi siempre y siempre, los estudiantes en cambio responden en más de un 50\% que nunca o a veces. En relación a la si los docentes dan retroalimentación a sus estudiantes, el 100\% de los profesores manifiestan que casi siempre y siempre, pero más del $45 \%$ de los estudiantes perciben que nunca o a veces.

\section{Conclusiones}

Este estudio demuestra que la organización, metodología de trabajo, motivación y evaluación tienen una correlación positiva. Es decir si mejora alguno de estos aspectos mejoran los demás. Sin embargo, hay unas relaciones más fuertes y otras más débiles, en razón de lo cual, habrá que hacer una indagación de cuáles son los motivos de estas fortalezas y debilidades. La percepción positiva de la metodología de trabajo se correlaciona mucho con la percepción tenida en la motivación y evaluación. Se evidencia también que una percepción positiva en

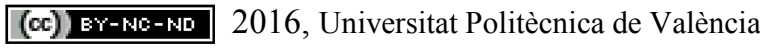

Congreso In-Red (2016) 
la motivación se relaciona mucho con una percepción positiva de la evaluación. Sin embargo, la organización dentro del aula no influye tanto en estos aspectos. Se demuestra que aunque se tenga una buena organización, la percepción metodológica no tiene porqué ser buena globalmente (tanto en estudiantes como en docentes, y considerando conjuntamente las dos escuelas).

Se ha demostrado que la valoración de los estudiantes y docentes es significativamente diferente principalmente en las metodologías de estudio aplicadas, estrategias de motivación y las estrategias de evaluación. Por ejemplo, en facilitar espacios para la discusión con sus estudiantes, mayor atención durante la ejecución de una tarea, en facilitar fuentes de estudio distintos al libro pauta. Se evidencia que existe una escasa dedicación individual de los docentes con sus alumnos; también es escaso el empleo de materiales impresos diferentes al libro de texto (libro guía).

No hay diferencias de opinión entre los encuestados de carreras distintas, ya sea docente o estudiante, hombre o mujer. Se evidencia que los docentes utilizan metodologías tradicionales (clase magistral), sin una evaluación integrada del proceso de aprendizaje (gestión de aula, tutorías y exámenes parciales) y no centrada en el estudiante mediante la reflexión, para alcanzar los mejores resultados en el aprendizaje. Hemos evidenciado escasa aplicación de metodologías de enseñanza/aprendizaje activas. Estas metodologías de trabajo aumentan la motivación de los estudiantes y, por tanto, aumentan las posibilidades de su aprendizaje.

Se demuestra la aplicabilidad del Análisis de Componentes principales como técnica de evaluación de la apreciación de las técnicas educativas. Esta técnica permite tratar máltiples variables de forma simultánea, sin ser ninguna determinante. En relación a los aspectos detectados con esta técnica se realizan recomendaciones.

\section{Recomendaciones}

- Mejorar los espacios de atención individual al estudiante.

- Facilitar fuentes de estudio distintos al libro pauta, entre otros: artículos científicos, estudios de prospección, fuentes de información, bibliotecas físicas y virtuales, entre otras.

- Poner mayor atención durante la ejecución de una tarea, en razón de que los docentes perciben de forma distinta (98\%) que sus alumnos $(50 \%)$, en relación a si los docentes indican cómo pueden corregirla, para que el empeño que pone el profesor en este aspecto, sea una percepción positiva de los estudiantes de las dos escuelas: arquitectura e ingeniería.

- Señalar los objetivos que alcanzarán al finalizar el tema y las estrategias que ayuden a los estudiantes a realizar sus actividades.

(cc) ) EY-NG-ND 2016, Universitat Politècnica de València 
- Hacer uso de diferentes metodologías para impartir las clases (acordes a los temas tratados) que ayuden a la clase magistral a cumplir su cometido, esto es que los estudiantes adquieran los conocimientos impartidos.

- Cambiar el modelo gestión en el aula y de tutoría actuales, por una tutoría individual, bajo acuerdo si el estudiante la requiere. Resolver ejercicios.

- Revisar el sistema de evaluación que usan los docentes, para que detecten el grado de adquisición de conocimientos de sus alumnos sobre la asignatura tratada.

- Señalar a los estudiantes el modo específico en que deben estudiar para una tarea evaluadora o un examen.

- Hacer que completen los estudiantes los ejercicios no terminados en clases o problemas en hojas, libros de trabajo o de texto.

- Determinar y dar a conocer el avance de los estudiantes.

- Dar retroalimentación a los estudiantes.

\section{Referencias}

ANTÓN-ARES, P. (2005). Motivación del profesorado universitario para la aplicación de las propuestas metodológicas derivadas de la utilización de las tecnologías de la información y de la comunicación en la docencia. Revista Latinoamericana de Tecnología Educativa, 4(1), 101-110.

DE MIGUEL-DÍAZ, M. (2005). Cambio de paradigma metodológico en la Educación Superior Exigencias que conlleva. Cuadernos de Integración Europea \#2 (16-27). Valencia: Universidad de Valencia. Recuperado de http://cde.uv.es/documents/2005-CIE-02.pdf

GARCÍA-BENAU, M., SIERRA-GARCÍA, L., y ZORIO-GRIMA, A. (2012). La verificación de la memoria de sostenibilidad en un contexto Europeo. Revista Globalización, Competitividad y Gobernabilidad, 6(2): 66-80. doi:10.3232/GCG.2012.V6.N2.04

GEORGE, D., y MALLERY, P. (2003). SPSS for Windows step by step: A simple guide and reference. 11.0 update (4th ed.). Boston: Allyn y Bacon.

GUBA, E.G., y LINCOLN, Y.S. (1989). Fourth generation evaluation. Newburg Park (California): Sage Publications.

JOVER, D. (2011). El poder de la educación para transformar la sociedad ¿cómo promover una educación que cuestione y transforme los modelos actuales?. I Congreso Internacional Educación y Soberanía Alimentaria, Barcelona, 13/10/2011. Recuperado de https://www.educacionsinfronteras.org/files/515984

LUENGO-NAVAS, J., LUZÓN-TRUJILLO, A., y TORRES-SÁNCHEZ, H. (Coords). (2008). Las reformas educativas basadas en el enfoque por competencias: Una visión comparada. Profesorado: Revista de currículum y formación del profesorado, 12(3), 1-10, monográfico.

MOURSHED, M., CHIJIOKE, C., y BARBER M. (2010). How the world's most improved school systems keep getting better. London: McKinsey y Company. Recuperado de http://hub.mspnet.org/index.cfm/22017

SÁNCHEZ-GARCÍA, J.G, GARCÍA-CHAMIZO, J.M., MORA-MORA, H., y SIGNES-PONTS, M.T. (2007). Metodologías docentes ECTS para la asignatura Informática Básica. V Jornadas de Investigación en Docencia Universitaria. Alicante, 04/06/2007. Recuperado en http://www.eduonline.ua.es/jornadas2007/comunicaciones/3E5.pdf

SÁEZ, F.J. (2000). La opinión de los estudiantes universitarios sobre el método docente de las facultades de ciencias. Revista de investigación educativa,1(18), 37-45.

SUEIRO, E. (2014). Comunicar o No Ser; escuchar y gestionar percepciones: el nuevo liderazgo. Madrid: Ed. Rasche.

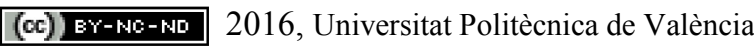

Congreso In-Red (2016) 
Evaluación Bidireccional del Proceso Enseñanza-Aprendizaje Universitario Aplicando Análisis de Componentes Principales

TEJEDOR-TEJEDOR, F.J. (2003). Un modelo de evaluación del profesorado universitario. Revista de Investigación Educativa, 21(1):157-182.

UNE-66181:2008. (2008). Gestión de la calidad. Calidad de la Formación Virtual. Madrid: Asociación Española de Normalización y Certificación (AENOR).

VASILACHIS DE GIALDINO, I. (Coord.). (2009). Estrategias de investigación cualitativa. Barcelona: Gedisa Editorial.

VÉLAZ DE MEDRANO, C., y VAILlANT, D. (Coords.). (2009). Aprendizaje y desarrollo profesional docente. Madrid: Fundación Santillana.

ZABALZA, M.A. (2004). Innovación en la enseñanza universitaria. Contextos Educativos, 6-7, $113-$ 136.

ZABALZA, M.A. (2012). Las competencias en la formación del profesorado: de la teoría a las propuestas prácticas. Tendencias pedagógicas, 20, 5-32.

(cc) EY-NG-ND 2016, Universitat Politècnica de València 